\title{
Computable solution of fractional kinetic equations using Mathieu-type series
}

\author{
Owais Khan ${ }^{1 *}$ (D), Nabiullah Khan ${ }^{1}$, Dumitru Baleanu ${ }^{2,3}$ and Kottakkaran Sooppy Nisar ${ }^{4}$
}

"Correspondence:

owkhan05@gmail.com

'Department of Applied

Mathematics, Aligarh Muslim

University, Aligarh, India

Full list of author information is

available at the end of the article (c) The Author(s) 2019. This article is distributed under the terms of the Creative Commons Attribution 4.0 International License (http://creativecommons.org/licenses/by/4.0/), which permits unrestricted use, distribution, and reproduction in any medium, provided you give appropriate credit to the original author(s) and the source, provide a link to the Creative Commons license, and indicate if changes were made.

\begin{abstract}
The Mathieu series appeared in the study of elasticity of solid bodies in the work of Émile Leonard Mathieu. Since then numerous authors have studied various problems arising from the Mathieu series in several diverse ways. In this line, our aim is to study the solution of fractional kinetic equations involving generalized Mathieu-type series. The generality of this series will help us to deduce results related to a fractional kinetic equation involving another form of Mathieu series. To obtain the solution, we use the Laplace transform technique. Besides, a graphical representation is given to observe the behavior of the obtained solutions.
\end{abstract}

MSC: Primary 26A33; secondary 33A10; 33E20; 44A10

Keywords: Generalized fractional kinetic equation; Mathieu-type series; Laplace transform; Sumudu transform

\section{Introduction and preliminaries}

Fractional calculus (FC) is a useful mathematical tool to study fractional order integrals and derivatives. The fractional calculus has been developed and used in different areas of science and engineering. The concept of fractional differential equations and their applications have played a significant role in many diverse fields such as applied science, physics, biology, chemistry, and engineering. The kinetic equations designate a system of differential equations, which describe the rate of change of the chemical composition of a star for each order in terms of the reaction rates for production and destruction. During the last several decades, fractional kinetic equations in various forms have been broadly and usefully employed when describing and solving several important problems of physics and astrophysics (see, e.g., [2, 7-11, 13-16, 20, 24-29, 31] and the references therein). The special functions and their applications appear in the solutions of fractional integral and differential equations and are related to comprehensive problems in the several areas of mathematics and mathematical physics. In view of the effectiveness and great importance of the fractional kinetic equations in certain astrophysical problems, the authors develop a generalized form of the fractional kinetic equations along with Mathieu-type series. The broad generality of Mathieu-type series will allow us to deduce many special cases of the main results. 
In [14], Haubold and Mathai explored the kinetic equation describing the rate of change of detraction, production, and reaction, which is given as

$$
\frac{d N}{d t}=p\left(N_{t}\right)-d\left(N_{t}\right)
$$

where $d=d(N)$ is the rate of destruction, $p=p(N)$ the rate of production, $N=N(t)$ the rate of reaction, and $N_{t}$ denotes the function given by $N_{t}\left(t^{*}\right)=N\left(t-t^{*}\right), t>0$. A particular case of (1), when special fluctuations or inhomogeneities in quantity $N(t)$ are decayed, is given by the equation

$$
\frac{d N}{d t}=-c_{i} N_{i}(t)
$$

with basic condition $N_{i}(t=0)=N_{0}$ describing the variety of density of species $i$ at time $t=0$ and $c_{i}>0$. Neglecting index $i$ and integrating, (2) becomes

$$
N(t)-N_{0}=-c_{00} D_{t}^{-1} N(t)
$$

where ${ }_{0} D_{t}^{-1}$ is the standard integral operator.

Further, a fractional generalization of (3) was given by Haubold and Mathai [14] in the following form:

$$
N(t)-N_{0}=-c^{v}{ }_{0} D_{t}^{-v} N(t)
$$

where ${ }_{0} D_{t}^{-v}$ represents the Riemann-Liouville integral operator defined as

$$
{ }_{0} D_{t}^{-\nu}=\frac{1}{\Gamma(\nu)} \int_{0}^{t}(-s+t)^{\nu-1} f(s) d s \quad(\Re(\nu)>0, t>0) .
$$

Saxena et al. [26] investigated solutions of three generalized forms of (4) in terms of the following generalized Mittag-Leffler function (see, e.g., [40]):

$$
E_{v, \mu}(z)=\sum_{r=0}^{\infty} \frac{z^{r}}{\Gamma(v r+\mu)} \quad(\Re(v)>0, \mu \in \mathbb{C}) .
$$

We here recall just one of them (see [26, Theorem 2]). The equation

$$
N(t)-N_{0} t^{\mu-1} E_{v, \mu}\left(-d^{v} t^{\nu}\right)=-c^{v}{ }_{0} D_{t}^{-v} N(t)
$$

is solved by

$$
N(t)=N_{0} \frac{t^{\mu-\nu-1}}{c^{\nu}-d^{\nu}}\left[E_{v, \mu-\nu}\left(-d^{\nu} t^{\nu}\right)-E_{v, \mu-\nu}\left(-d^{\nu} t^{\nu}\right)\right]
$$

where $\mu, v, c, d \in \mathbb{R}^{+} ; c \neq d$.

Sexena et al. [28] investigated the following generalized fractional kinetic equation:

$$
N(t)-N_{0} t^{\mu-1} E_{v, \mu}^{\gamma}\left(-d^{v} t^{\nu}\right)=-c^{\nu}{ }_{0} D_{t}^{-v} N(t) \quad\left(\mu, v, \gamma, c \in \mathbb{R}^{+}\right),
$$


and its solution is

$$
N(t)=N_{0} t^{\mu-1} E_{\nu, \mu}^{\gamma+1}\left(-c^{\nu} t^{\nu}\right)
$$

where $E_{v, \mu}^{\gamma}(z)$ is an extension of the generalized Mittag-Leffler function (6) (see [23]):

$$
E_{v, \mu}^{\gamma}(z)=\sum_{r=0}^{\infty} \frac{(\gamma)_{r} z^{r}}{\Gamma(\nu r+\mu)} \quad(\Re(v)>0 ; \mu, \gamma \in \mathbb{C})
$$

and $(\eta)_{\varepsilon}$ denotes the Pochhammer symbol which is defined (for $\eta, \varepsilon \in \mathbb{C}$ ) in terms of the gamma function $\Gamma$ as

$$
(\eta)_{\varepsilon}:=\frac{\Gamma(\eta+\varepsilon)}{\Gamma(\eta)}= \begin{cases}1 & (\varepsilon=0 ; \eta \in \mathbb{C} \backslash\{0\}), \\ \eta(\eta+1) \cdots(\eta+n-1) & (\varepsilon=n \in \mathbb{N} ; \eta \in \mathbb{C}) .\end{cases}
$$

Saxena and Kalla [25] investigated the following fractional kinetic equation:

$$
N(t)-N_{0} f(t)=-c^{v}{ }_{0} D_{t}^{-v} N(t) \quad\left(v, c \in \mathbb{R}^{+}\right),
$$

where $f \in L(0, \infty)$. Applying the Laplace transform (LT) to (13), we have

$$
L[N(t): s]=N_{0} \frac{F(s)}{c^{v} s^{-v}+1}=N_{0} F(s)\left(\sum_{n \geq 0}\left(-c^{v}\right)^{n} s^{-n v}\right) \quad\left(n \in N_{0},\left|\frac{c}{s}\right|\right) .
$$

Suppose that $f(t)$ is a real- (or complex-) valued function of the (time) variable $t>0$ and $s$ is real or complex parameter. The Laplace transform of the function $f(t)$ is defined by

$$
\begin{aligned}
F(s) & =L\{N(t) ; s\}=\int_{0}^{\infty} e^{-s t} f(t) d t \quad(\Re(s)>0) \\
& =\lim _{\varepsilon \rightarrow \infty} \int_{0}^{\varepsilon} e^{-s t} f(t) d t,
\end{aligned}
$$

whenever the limit exits (as a finite number). The convolution of two functions $f(t)$ and $g(t)$, which are defined for $t>0$, plays an important role in a number of different physical applications. The Laplace convolution of functions $f(t)$ and $g(t)$ is given by the following integral:

$$
(f * g)(t)=\int_{0}^{t} f(u) g(1-u) d u=(g * f)(t)
$$

which exists if the functions $f$ and $g$ are at least piecewise continuous. One of the most significant properties possessed by the convolution in connection with the Laplace transform is that the Laplace transform of the convolution of two functions is the product of their transforms (see, e.g., [20, 30, 32, 33]).

The Laplace Convolution Theorem Iff and $g$ are piecewise continuous on $[0, \infty)$ and of exponential order $\alpha$ when $t \rightarrow \infty$, then

$$
L\{(f * g)(t): s\}=L\{f(t): s\} \cdot L\{g(t): s\} \quad(\Re(s)>0) .
$$


We find

$$
\begin{aligned}
L\left\{{ }_{0} D_{t}^{-v} f(t): s\right\} & =\frac{1}{\Gamma(\nu)} L\left\{t^{\nu-1} * f(t): s\right\} \\
& =\frac{1}{\Gamma(v)} L\left\{t^{\nu-1}: s\right\} L\{f(t): s\}=\frac{1}{s^{\nu}} L\{f(t): s\},
\end{aligned}
$$

by using the following well-known identity:

$$
\begin{aligned}
L\left\{t^{\nu}: s\right\} & =\frac{\Gamma(v+1)}{s^{\nu+1}} \\
& \Longleftrightarrow L^{-1}\left(\frac{1}{s^{\nu+1}}\right)=\frac{t^{\nu}}{\Gamma(v+1)},
\end{aligned}
$$

in which $L^{-1}$ denotes the inverse Laplace transform.

Recently, many papers investigated the solution of generalized fractional kinetic equations involving a variety of special functions by using Laplace and Sumudu transformations. For instance, for generalized fractional kinetic equations with $f(t)$ in (13) replaced by certain forms of generalized Mittag-Leffler function (11), Saxena et al. [29] presented their corresponding solutions. Moreover, Chaurasia and Kumar [7] investigated the solution of generalized fractional kinetic equations involving $\mathrm{M}$-series, and Choi and Kumar [9] gave their corresponding solutions involving Aleph function. Furthermore, Agarwal et al. [1,2] investigated solutions of fractional kinetic equations with $f(t)$ in (13) replaced by certain forms of $k$-Mittag-Leffler function and $k$-Bessel function. Subsequently, Nisar and Qi [22] presented solution of fractional kinetic equations with $f(t)$ in (13) replaced by certain forms of generalized $k$-Bessel function.

The following familiar infinite series was introduced by Mathieu [21]:

$$
S(l)=\sum_{k=1}^{\infty} \frac{2 k}{\left(k^{2}+l^{2}\right)} \quad\left(l \in \mathbb{R}^{+}\right) .
$$

An integral representation of (21) is given by (see [12])

$$
S(l)=\frac{1}{l} \int_{0}^{\infty} \frac{t \sin (l t)}{e^{t}-1} d t
$$

Numerous interesting problems and solutions handling integral representations and related to the following generalization of the Mathieu series with fractional power

$$
S_{\mu}(l)=\sum_{k=1}^{\infty} \frac{2 k}{\left(k^{2}+l^{2}\right)^{\mu}} \quad\left(l \in \mathbb{R}^{+} ; \mu \leq 1\right)
$$

can be found in the works by Cerone and Lenard [6] as well as Tomovski and Trencevski [37]. Extending the work of Cerone and Lenard [6], Srivastava and Tomovski [34] defined a family of generalized Mathieu series

$$
S_{\mu}^{(\rho, \sigma)}(l, d)=S_{\mu}^{(\rho, \sigma)}\left(l,\left\{d_{k}\right\}_{k=1}^{\infty}\right)=\sum_{k=1}^{\infty} \frac{2 d_{k}^{\sigma}}{\left(d_{k}^{\rho}+l^{2}\right)^{\mu}} \quad\left(l, d, \rho, \sigma, \mu \in \mathbb{R}^{+}\right),
$$


where the positive sequence $d=\left\{d_{k}\right\}_{k=1}^{\infty}=\left\{d_{1}, d_{2}, \ldots\right\}\left(\lim _{k \rightarrow \infty} d_{k}=\infty\right)$ is such that the infinite series $\sum_{k=1}^{\infty} \frac{1}{d_{k}^{\mu \rho-\sigma}}$ is convergent.

In the sequel, Tomovski and Mehrez [35] proposed a generalization of definition (24) in the following power series:

$$
\begin{aligned}
S_{\mu, \tau}^{(\rho, \sigma)}(l, d ; u) & =S_{\mu, \tau}^{(\rho, \sigma)}\left(l,\left\{d_{k}\right\}_{k=1}^{\infty} ; u\right) \\
& =\sum_{k=1}^{\infty} \frac{2 d_{k}^{\sigma}(\tau)_{k}}{\left(d_{k}^{\rho}+l^{2}\right)^{\mu}} \frac{u^{k}}{k !} \quad\left(l, d, \rho, \sigma, \mu \in \mathbb{R}^{+} ;|u| \leq 1\right) .
\end{aligned}
$$

Evidently, the case $d_{k}=k, \rho=2, \sigma=1=\tau$ and $\mu$ replaced with $\mu+1$ corresponds to the Mathieu series defined by Tomovski and Pogány [36]:

$$
S_{\mu+1,1}^{(2,1)}(l, k ; u)=S_{\mu}(l ; u)=\sum_{k=1}^{\infty} \frac{2 k u^{k}}{\left(k^{2}+l^{2}\right)^{\mu+1}} \quad\left(l, \mu \in \mathbb{R}^{+} ;|u| \leq 1\right) .
$$

Due to the great importance of fractional kinetic equations involving special functions, in this paper, we aim to investigate solution of generalized fractional kinetic equations (13) with $f(t)$ replaced by several generalized Mathieu-type series, by mainly using the Laplace and Sumudu transforms. The results presented here, being general, are also shown to reduce to fractional kinetic equations involving simpler special functions. The manuscript is organized as follows. In Sect. 2, the solution of fractional kinetic equations involving Mathieu-type series is established, and in Sect. 3, a graphical interpretation and nature of the solution are discussed. Section 4 is focused on some examples, and in Sect. 5 concluding remarks are given.

\section{Solution of fractional kinetic equations}

In this section, we obtain the solution of generalized fractional kinetic equation (13) involving generalized Mathieu-type series (25) by applying the Laplace transform technique.

Theorem 2.1 For all $v, c, h>0 ; c \neq h$, if $\mu, \rho, \sigma, \tau, l \in \mathbb{R}^{+},|h t| \leq 1$, then equation

$$
N(t)-N_{0} S_{\mu, \tau}^{(\rho, \sigma)}\left(l,\left\{d_{k}\right\}_{k=1}^{\infty} ; h t\right)=-c^{\nu}{ }_{0} D_{t}^{-\nu} N(t)
$$

has the solution

$$
N(t)=N_{0} \sum_{k=1}^{\infty} \frac{2 d_{k}^{\sigma}(\tau)_{k}}{\left(d_{k}^{\rho}+l^{2}\right)^{\mu}}(h t)^{k} E_{v, k+1}\left(-c^{\nu} t^{\nu}\right) .
$$

Proof Applying the Laplace transform to (27) and using (25) and (18) gives

$$
N^{*}(s)=N_{0}\left(\int_{0}^{\infty} e^{-s t} \sum_{k=1}^{\infty} \frac{2 d_{k}^{\sigma}(\tau)_{k}}{\left(d_{k}^{\rho}+l^{2}\right)^{\mu}} \frac{(h t)^{k}}{k !}\right) d t-c^{\nu} s^{-v} N^{*}(s)
$$

where $N^{*}(s)=L\{N(t) ; s\}$. 
Under the given assumptions, computing the integral in (29) term by term and using $L\left\{t^{\lambda} ; s\right\}=\frac{\Gamma(\lambda+1)}{s^{\lambda+1}}$, we have

$$
\left(1+\left(\begin{array}{c}
c \\
s
\end{array}\right)^{v}\right) N^{*}(s)=N_{0} \sum_{k=1}^{\infty} \frac{2 d_{k}^{\sigma}(\tau)_{k}}{\left(d_{k}^{\rho}+l^{2}\right)^{\mu}} \frac{h^{k}}{k !} \frac{\Gamma(1+k)}{s^{1+k}}
$$

Employing the geometric series expansion of $\left(1+\left(\frac{c}{s}\right)^{v}\right)^{-1}$ for $c<|s|$, we have

$$
\begin{aligned}
N^{*}(s)= & N_{0} \sum_{k=1}^{\infty} \frac{2 d_{k}^{\sigma}(\tau)_{k}}{\left(d_{k}^{\rho}+l^{2}\right)^{\mu}} \frac{h^{k}}{s^{1+k}} \\
& \times \sum_{r=0}^{\infty}(-1)^{r}\left(\frac{c}{s}\right)^{v r} .
\end{aligned}
$$

Taking the inverse Laplace transform and applying the relation $L^{-1}\left\{s^{-v} ; t\right\}=\frac{t^{v-1}}{\Gamma(v)}, \mathfrak{R}(v)>0$, we get

$$
\begin{aligned}
N(t) & =L^{-1}\left\{N^{*}(s) ; t\right\} \\
& =N_{0} \sum_{k=1}^{\infty} \frac{2 d_{k}^{\sigma}(\tau)_{k}}{\left(d_{k}^{\rho}+l^{2}\right)^{\mu}}(h t)^{k}\left\{\sum_{r=0}^{\infty} \frac{(-1)^{r}(c t)^{\nu r}}{\Gamma(\nu r+1+k)}\right\} \\
& =N_{0} \sum_{k=1}^{\infty} \frac{2 d_{k}^{\sigma}(\tau)_{k}}{\left(d_{k}^{\rho}+l^{2}\right)^{\mu}}(h t)^{k} E_{\nu, k+1}\left(-c^{\nu} t^{\nu}\right) .
\end{aligned}
$$

In view of relation (26), we state the following consequence of Theorem 2.1.

Corollary For all $v, c, h>0 ; c \neq h$, if $\mu, l \in \mathbb{R}^{+},|h t| \leq 1$, then equation

$$
N(t)-N_{0} S_{\mu, \tau}(l, k ; h t)=-c^{v}{ }_{0} D_{t}^{-v} N(t)
$$

has the solution

$$
N(t)=N_{0} \sum_{k=1}^{\infty} \frac{2 k}{\left(k^{2}+l^{2}\right)^{\mu+1}}(h t)^{k} E_{v, k+1}\left(-c^{v} t^{v}\right)
$$

Theorem 2.2 Assume that $\nu, c, w>0$ and $\mu, \rho, \sigma, \tau, l \in \mathbb{R}^{+},|w t| \leq 1$, then equation

$$
N(t)-N_{0} S_{\mu, \tau}^{(\rho, \sigma)}\left(l,\left\{d_{k}\right\}_{k=1}^{\infty} ; w t^{\nu}\right)=-\left[\sum_{r=1}^{n}\left(\begin{array}{l}
n \\
r
\end{array}\right) c^{\nu r}{ }_{0} D_{t}^{-v r}\right] N(t)
$$

has the solution

$$
N(t)=N_{0} t^{\alpha-1} \sum_{k=1}^{\infty} \frac{2 d_{k}^{\sigma}(\tau)_{k} \Gamma(v k+1)}{\left(d_{k}^{\rho}+l^{2}\right)^{\mu}} \frac{\left(w t^{\nu}\right)^{k}}{k !} E_{v, 1+v k}^{n}\left(-c^{\nu} t^{\nu}\right) .
$$

Proof Applying LT to both sides of (33) and then using (25) and (15), we get

$$
L\{N(t) ; s\}=N_{0} L\left\{S_{\mu, \tau}^{(\rho, \sigma)}\left(l,\left\{d_{k}\right\}_{k=1}^{\infty} ; w t^{\nu}\right) ; s\right\}-L\left\{\left[\sum_{r=1}^{n}\left(\begin{array}{l}
n \\
r
\end{array}\right) c_{0}^{\nu r} D_{t}^{-v r}\right] N(t) ; s\right\}
$$


which, upon solving for $N^{*}(s)$, yields

$$
\begin{aligned}
& N^{*}(s)-N_{0} \sum_{k=1}^{\infty} \frac{2 d_{k}^{\sigma}(\tau)_{k}}{\left(d_{k}^{\rho}+l^{2}\right)^{\mu}} \frac{w^{k}}{k !} \frac{\Gamma(1+v k)}{s^{1+v k}} \\
& =-\left[C_{1}\left(\frac{c}{s}\right)^{v}+{ }^{n} C_{2}\left(\frac{c}{s}\right)^{2 v}+\cdots+{ }^{n} C_{n}\left(\frac{c}{s}\right)^{n v}\right] N^{*}(s)=-\left(\frac{c}{s}\right)^{n v} N^{*}(s), \\
& N^{*}(s)=N_{0} \sum_{k=1}^{\infty} \frac{2 d_{k}^{\sigma}(\tau)_{k}}{\left(d_{k}^{\rho}+l^{2}\right)^{\mu}} \frac{w^{k}}{k !} \frac{\Gamma(1+v k)}{s^{1+v k}}\left(1+\left(\frac{c}{s}\right)^{\nu}\right)^{-n} .
\end{aligned}
$$

Employing the binomial formula

$$
(1-u)^{-\lambda}=\sum_{r=0}^{\infty} \frac{(\lambda)_{r}}{r !} u^{r}
$$

which obviously converges for all $|u|<1$, we have

$$
N^{*}(s)=N_{0} \sum_{k=1}^{\infty} \frac{2 d_{k}^{\sigma}(\tau)_{k}}{\left(d_{k}^{\rho}+l^{2}\right)^{\mu}} \frac{w^{k}}{k !} \frac{\Gamma(1+v k)}{s^{1+v k}}\left\{\sum_{j=0}^{\infty}(-1)^{j} \frac{(n)_{j}}{j !}\left(-c^{\nu} s^{-\nu}\right)^{j}\right\} .
$$

If we now take the inverse LT of (35) and use (11), after easy simplification, the result (34) readily follows.

In view of relation (26), we establish the following consequence of Theorem 2.2.

Corollary Assume that $v, \alpha, c, w>0$, and let $\mu, l \in \mathbb{R}^{+},|w t| \leq 1$, then equation

$$
N(t)-N_{0} t^{\alpha-1} S_{\mu}\left(l ; w t^{\nu}\right)=-\left[\sum_{r=1}^{n}\left(\begin{array}{l}
n \\
r
\end{array}\right) c^{\nu r}{ }_{0} D_{t}^{-v r}\right] N(t)
$$

has the solution

$$
N(t)=N_{0} \sum_{k=1}^{\infty} \frac{2 k \Gamma(v k+1)}{\left(k^{2}+l^{2}\right)^{\mu+1}} \frac{\left(w t^{\nu}\right)^{k}}{k !} E_{v, 1+\nu k}^{n}\left(-c^{\nu} t^{\nu}\right) .
$$

Theorem 2.3 For all $v>0, c>0, \mu, \rho, \sigma, \tau, l \in \mathbb{R}^{+},|t| \leq 1$, the equation

$$
N(t)-N_{0} S_{\mu, \tau}^{(\rho, \sigma)}\left(l,\left\{d_{k}\right\}_{k=1}^{\infty} ; c^{v} t^{\nu}\right)=-c^{v}{ }_{0} D_{t}^{-v} N(t)
$$

has the solution

$$
N(t)=N_{0} \sum_{k=1}^{\infty} \frac{2 d_{k}^{\sigma}(\tau)_{k} \Gamma(v k+1)}{\left(d_{k}^{\rho}+l^{2}\right)^{\mu}} \frac{\left(c^{v} t^{\nu}\right)^{k}}{k !} E_{v, v k+1}\left(-c^{v} t^{\nu}\right) .
$$

Proof This can be proved by the same procedure as in the proof of Theorem 2.1. So we omit all details.

Using relation (26), we state the following consequence of Theorem 2.3. 
Corollary For all $v>0, c>0, \mu, l \in \mathbb{R}^{+},|t| \leq 1$, the equation

$$
N(t)-N_{0} S_{\mu}\left(l ; c^{v} t^{v}\right)=-c^{v}{ }_{0} D_{t}^{-v} N(t)
$$

has the solution

$$
N(t)=N_{0} \sum_{k=1}^{\infty} \frac{2 k \Gamma(\nu k+1)}{\left(k^{2}+l^{2}\right)^{\mu+1}} \frac{\left(c^{\nu} t^{\nu}\right)^{k}}{k !} E_{v, \nu k+1}\left(-c^{\nu} t^{\nu}\right) .
$$

\section{Graphical representations}

Figures 1-5 represents the graph of solution (28) by considering some fractional values, and observe that, by choosing small values for $v$, the graph is increasing in nature, and if we choose larger values for $v$, then it is gradually decreasing. The graphical results confirm that the region of convergence of solutions depends continuously on the fractional parameter $v$. Hence, by observing the nature of the solutions for various parameters and time interval, it is concluded that $N(t)$ can be negative as well as positive. A similar observation can be obtained for the solutions (34) and (39).

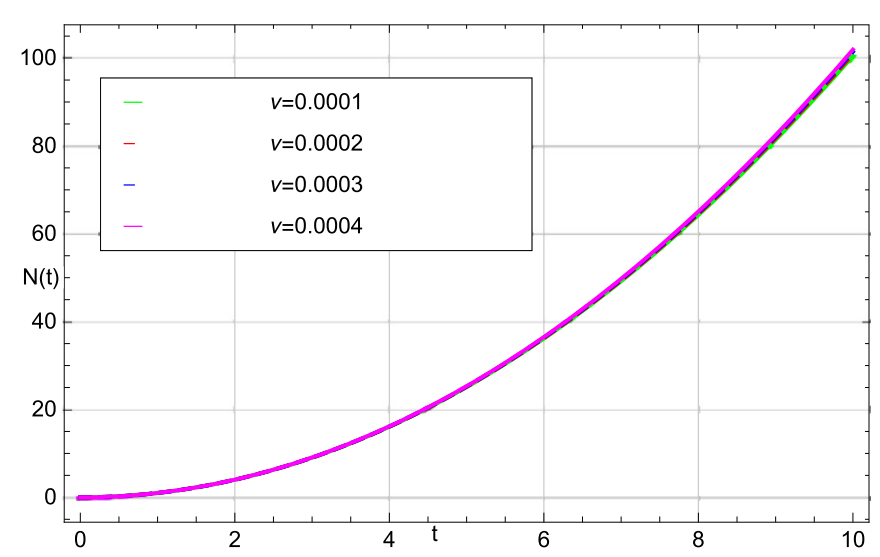

Figure 1 Solution (13) for $N(t)$ and $t$

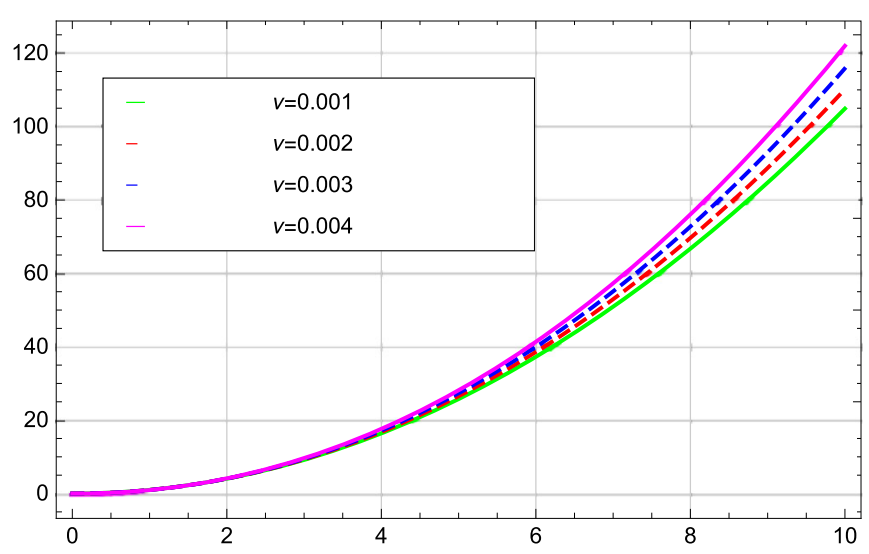

Figure 2 Solution (13) for $N(t)$ and $t$ 
Khan et al. Advances in Difference Equations

(2019) 2019:234

Page 9 of 13

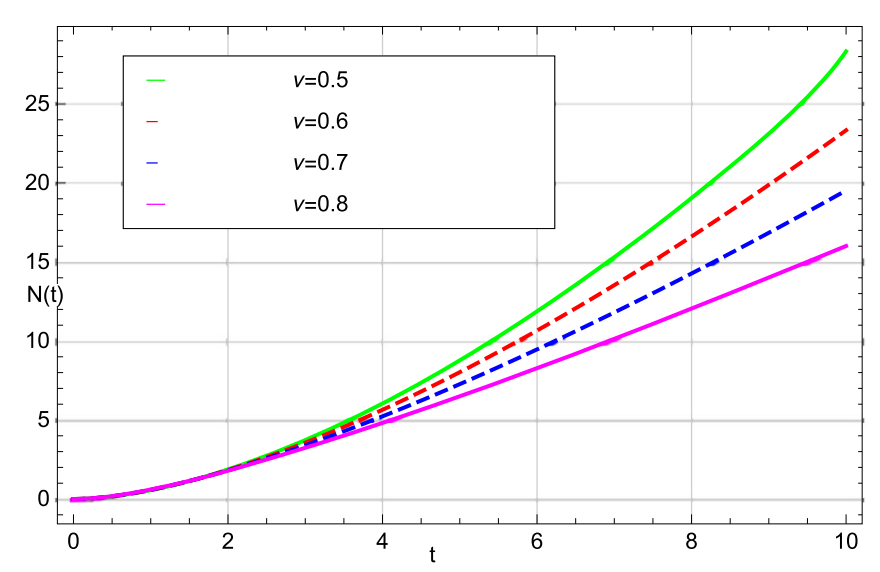

Figure 3 Solution (13) for $N(t)$ and $t$

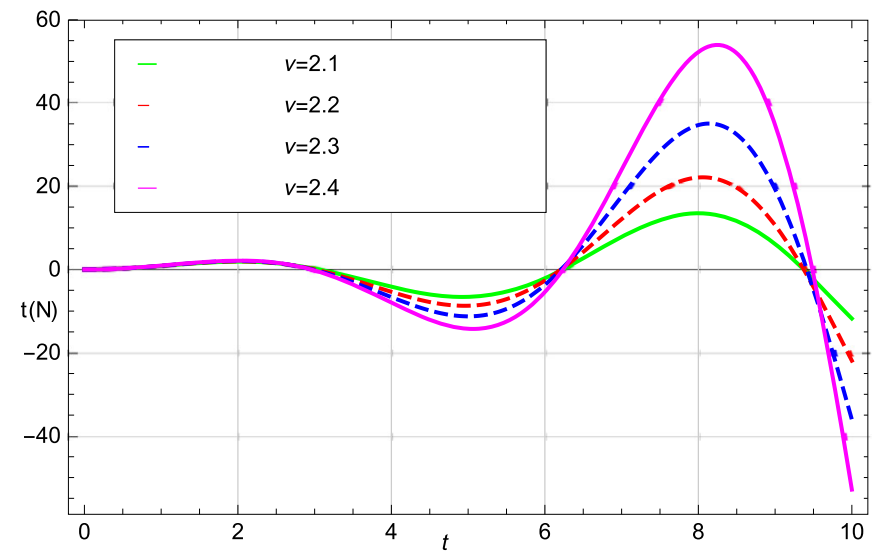

Figure 4 Solution (13) for $N(t)$ and $t$

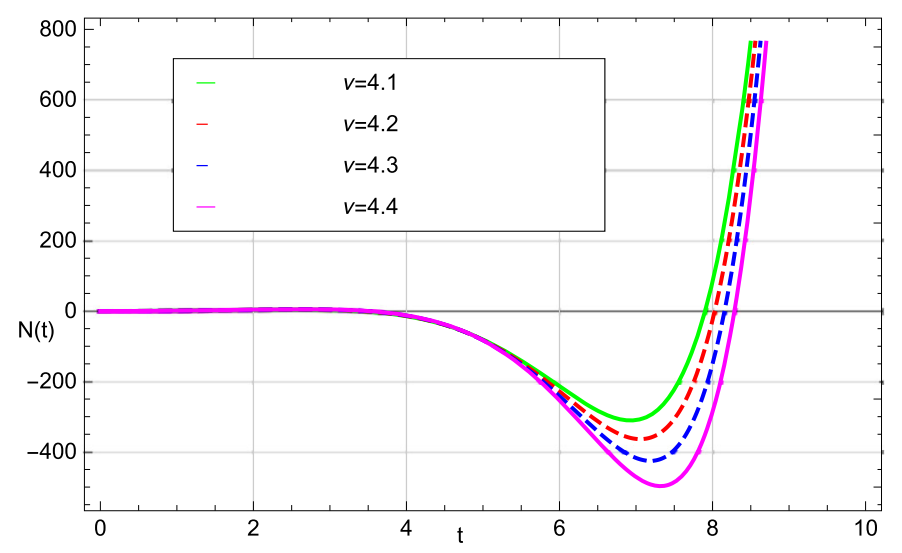

Figure 5 Solution (13) for $N(t)$ and $t$ 


\section{Example}

A detailed account of Mathieu-type series and their applications can be found in the monographs by various authors [21, 35-37]. An integral transform, called the Sumudu transform, was defined and studied by Watugala [38] to facilitate the process of solving differential and integral equations in the time domain, and for the use in various applications of system control engineering and applied mathematics. In $[3,4]$, some fundamental properties of the Sumudu transform are investigated. It turns out that the Sumudu transform has very special and useful properties, and it is useful in solving problems of science and control engineering governing kinetic equations. The Sumudu transform is defined on the set of functions

$$
A=\left\{f(t)\left|\exists M, \delta_{1}, \delta_{2},\right| f(t) \mid<M e^{|t| / \delta_{j}} \text { if } t \in(-1)^{j} \times[0, \infty)\right\},
$$

by

$$
G(u)=S(f(t) ; u)=\int_{0}^{\infty} e^{-t} f(u t) d t, \quad u \in\left(-\delta_{1}, \delta_{2}\right),
$$

where $M$ is a real finite number and $\delta_{1}$ and $\delta_{2}$ can be finite or infinite. For more details, see $[38,39]$.

By using the convolution theorem for the Sumudu transform [3-5], (5) can be written in the following form:

$$
S\left\{{ }_{0} D_{t}^{-v} f(t)\right\}=S\left\{\frac{t^{\nu-1}}{\Gamma(v)}\right\} \cdot S\{f(t)\}=u^{v} G(u) .
$$

It is easy to see that the Sumudu transform of the function $f(t)=t^{\varepsilon}$ is given by

$$
S[f(t)]=\int_{0}^{\infty}(t y)^{\varepsilon} e^{-t} d t=u^{\varepsilon} \Gamma(\varepsilon+1) \quad(\Re(\varepsilon)>-1) .
$$

The Laplace transform is a very potent mathematical tool which is used to solve diverse engineering and science problems. The Sumudu transform (which is not so well-known as the Laplace transform) was proposed in the early 1990s, and it has some interesting advantages over other integral transforms, especially regarding the "unity" feature which could become convenient when solving differential equations. As a comparison, the Sumudu transform is a simple variant of the Laplace transform. This paper focuses on the effectiveness of both transforms to obtain the solutions of fractional kinetic equations. The Sumudu transform is essentially identical with the Laplace transform. Given an initial $f(t)$, its Laplace transform $F(s)$ can be translated into the Sumudu transform $F_{s}(u)$ of $f$ by means of the relation

$$
S(u)=\frac{F\left(\frac{1}{u}\right)}{u},
$$

and its inverse is

$$
F(s)=\frac{S\left(\frac{1}{s}\right)}{s} .
$$


For more details about the Sumudu transform and its properties in comparison to the Laplace transform, the interested readers can check [17-19].

Due to the importance of the above observation, in this section, we first define the Sumudu transform of Mathieu-type series (25) and further determine the solution of fractional kinetic equations by applying Sumudu transform technique as given in the following Examples 4.1, 4.2, and 4.3.

The following integral gives the Sumudu transform of Mathieu-type series (25):

$$
\int_{0}^{\infty} e^{-t}(u t)^{\varepsilon-1} S_{\mu, \tau}^{(\rho, \sigma)}\left(l,\left\{d_{k}\right\}_{k=1}^{\infty} ; w(u t)^{\nu}\right) d t=u^{\varepsilon-1} \sum_{k=1}^{\infty} \frac{2 d_{k}^{\sigma}(\tau)_{k}}{\left(d_{k}^{\rho}+l^{2}\right)^{\mu}} \frac{\Gamma(\varepsilon+v k)}{k !}(u w)^{k},
$$

where $\Re(u)>|w|^{-1 / \Re(v)}, \Re(\varepsilon)>0, u \in\left(-\delta_{1}, \delta_{2}\right),|f(t)|<M e^{|t| \mid \delta_{j}}$ and $l, d, \rho, \sigma, \mu \in \mathbb{R}^{+}$.

Using the same procedure of analysis as in Theorems 2.1,2.2, and 2.3, we can find the solutions of generalized fractional kinetic equations involving Mathieu-type series, which are given in the following three examples.

Example 4.1 If $\varepsilon, v, c, h>0, \Re(s)>0$ with $|s|<c^{-1}, c \neq h$ and $\mu, \rho, \sigma, \tau, l \in \mathbb{R}^{+}$, then the solution of the generalized fractional kinetic equation

$$
N(t)-N_{0} t^{\varepsilon-1} S_{\mu, \tau}^{(\rho, \sigma)}\left(l,\left\{d_{k}\right\}_{k=1}^{\infty} ; h t^{\nu}\right)=-c^{\nu}{ }_{0} D_{t}^{-\nu} N(t)
$$

is given by

$$
N(t)=N_{0} t^{\varepsilon-2} \sum_{k=1}^{\infty} \frac{2 d_{k}^{\sigma}(\tau)_{k} \Gamma(\nu k+\varepsilon)}{\left(d_{k}^{\rho}+l^{2}\right)^{\mu}} \frac{\left(c^{\nu} t^{\nu}\right)^{k}}{k !} E_{v, \varepsilon+v k-1}\left(-c^{\nu} t^{\nu}\right) .
$$

Example 4.2 If $v, c, w>0$ and $\mu, \rho, \sigma, \tau, l \in \mathbb{R}^{+} ;|s|<c^{-1}, c \neq w$, then the solution of the generalized fractional kinetic equation

$$
N(t)-N_{0} t^{\varepsilon-1} S_{\mu, \tau}^{(\rho, \sigma)}\left(l,\left\{d_{k}\right\}_{k=1}^{\infty} ; w t^{\nu}\right)=-\left[\sum_{r=1}^{n}\left(\begin{array}{l}
n \\
r
\end{array}\right) c^{\nu r}{ }_{0} D_{t}^{-v r}\right] N(t)
$$

is given by

$$
N(t)=N_{0} t^{\varepsilon-2} \sum_{k=1}^{\infty} \frac{2 d_{k}^{\sigma}(\tau)_{k} \Gamma(v k+\varepsilon)}{\left(d_{k}^{\rho}+l^{2}\right)^{\mu}} \frac{\left(w t^{\nu}\right)^{k}}{k !} E_{v, v k+\varepsilon-1}^{n}\left(-c^{\nu} t^{\nu}\right) .
$$

Example 4.3 If $v>0, c>0, \mu, \rho, \sigma, \tau, l \in \mathbb{R}^{+}$and $|s|<c^{-1}$, then the solution of the generalized fractional kinetic equation

$$
N(t)-N_{0} t^{\varepsilon-1} S_{\mu, \tau}^{(\rho, \sigma)}\left(l,\left\{d_{k}\right\}_{k=1}^{\infty} ; c^{\nu} t^{\nu}\right)=-c^{\nu}{ }_{0} D_{t}^{-\nu} N(t)
$$

is given by

$$
N(t)=N_{0} t^{\varepsilon-2} \sum_{k=1}^{\infty} \frac{2 d_{k}^{\sigma}(\tau)_{k} \Gamma(v k+\varepsilon)}{\left(d_{k}^{\rho}+l^{2}\right)^{\mu}} \frac{\left(c^{\nu} t^{\nu}\right)^{k}}{k !} E_{v, v k+\varepsilon-1}\left(-c^{\nu} t^{\nu}\right) .
$$




\section{Conclusion}

In the current study, we have obtained the solution of generalized fractional kinetic equations involving generalized Mathieu-type series with the help of Laplace and Sumudu transform techniques. The results of Sect. 2 are general in character and are likely to find certain applications in the theory of fractional calculus and special functions. From the graphical analysis, we conclude that $N(t)>0$ or $N(t)<0$ for distinct values of the parameters. By suitably specializing the values of the parameters of generalized Mathieu-type series (25), our main results can yield several new solutions of generalized fractional kinetic equations corresponding to the Mathieu series given by various authors $[6,21,34$, 37]. Therefore, the investigated results in this paper would at once give many results involving diverse special functions occurring in the problems of astrophysics, mathematical physics, and engineering.

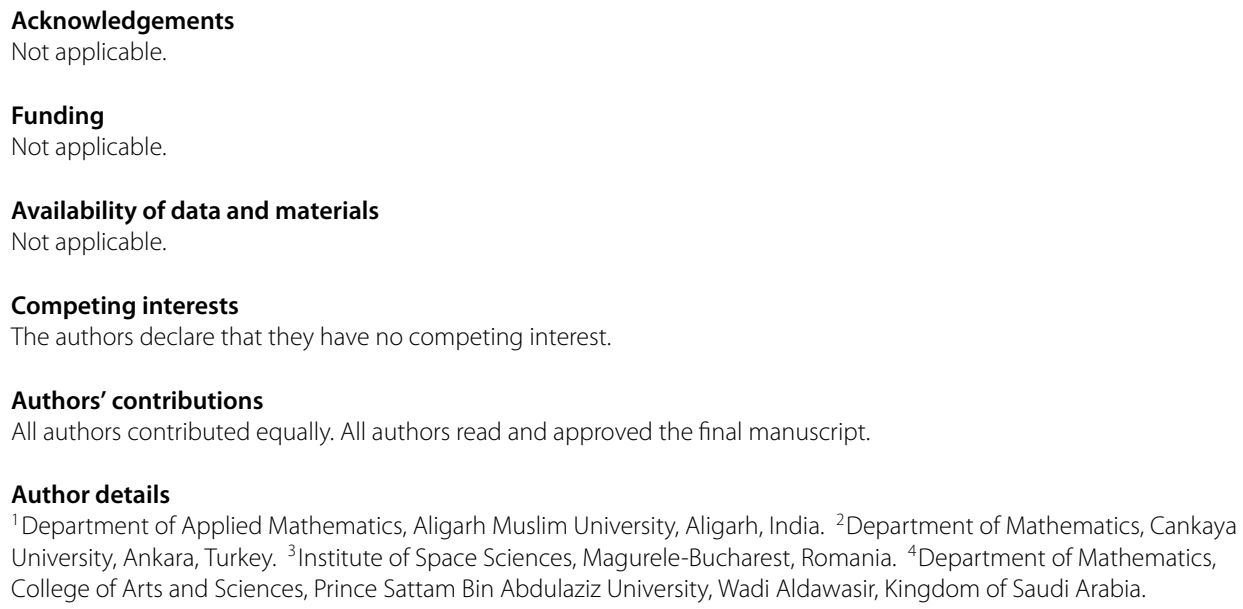

\section{Publisher's Note}

Springer Nature remains neutral with regard to jurisdictional claims in published maps and institutional affiliations.

Received: 7 February 2019 Accepted: 3 June 2019 Published online: 14 June 2019

References

1. Agarwal, P., Chand, M., Baleanu, D., O'Regan, D., Jain, S.: On the solution of certain fractional kinetic equations involving k-Mittag-Leffler function. Adv. Differ. Equ. 2018, 249 (2018) https://doi.org/10.1186/s13662-018-1694-8

2. Agarwal, P., Chand, M., Singh, G.: Fractional kinetic equations involving generalized $k$-Bessel function via Sumudu transform. Alex. Eng. J. 55(4), 3053-3059 (2016)

3. Asiru, M.A.: Sumudu transform and the solution of integral equations of convolution type. Int. J. Math. Educ. Sci. Technol. 32(6), 906-910 (2001)

4. Belgacem, F.B.M., Karaballi, A.A.: Sumudu transform fundamental properties investigations and applications. J. Appl. Math. Stoch. Anal. 2006, Article ID 91083 (2006) https://doi.org/10.1155/JAMSA/2006/91083

5. Belgacem, F.B.M., Karaballi, A.A., Kalla, S.L.: Analytical investigations of the Sumudu transform and applications to integral production equations. Math. Probl. Eng. 3, 103-118 (2003)

6. Cerone, P., Lenard, C.T.: On integral forms of generalized Mathieu series. JIPAM. J. Inequal. Pure Appl. Math. 4, Article ID $100(2003)$

7. Chaurasia, V.B.L., Kumar, D.: On the solution of generalized fractional kinetic equation. Adv. Stud. Theor. Phys. 4, 773-780 (2010)

8. Chaurasia, V.B.L., Pandey, S.C.: On the new computable solution of the generalized fractional kinetic equations involving the generalized function for the fractional calculus and related functions. Astrophys. Space Sci. 317, 213-219 (2008)

9. Choi, J., Kumar, D.: Solutions of generalized fractional kinetic equations involving Aleph functions. Math. Commun. 20, 113-123 (2015)

10. Dorrego, G., Kumar, D.: A generalization of the kinetic equation using the Prabhakar-type operators. Honam Math. J. 39(3), 401-416 (1017)

11. Dutta, B.K., Arora, L.K., Borah, J.: On the solution of fractional kinetic equation. Gen. Math. Notes 6, 40-48 (2011)

12. Emersleben, O.: Über die Reihe. Math. Ann. 125, 165-171 (1952) 
13. Gupta, V.G., Sharma, B., Belgacem, F.B.M.: On the solutions of generalized fractional kinetic equations. Appl. Math. Sci. 5(17-20), 899-910 (2011)

14. Haubold, H.J., Mathai, A.M.: The fractional kinetic equation and thermonuclear functions. Astrophys. Space Sci. 273 , 53-63 (2000)

15. Kamarujjama, M., Khan, N.U., Khan, O.: The generalized p-k-Mittag-Leffler function and solution of fractional kinetic equations. J. Anal. in press. https://doi.org/10.1007/s41478-018-0160-z

16. Kamarujjama, M., Khan, O.: Computation of new class of integrals involving generalized Galue type Struve function. J. Comput. Appl. Math. 351, 228-236 (2019)

17. Kilicman, A., Eltayeb, H.: Some remarks on the Sumudu and Laplace transforms and applications to differential equations. ISRN Appl. Math. 2012, Article ID 591517 (2012) https://doi.org/10.5402/2012/591517

18. Kilicman, A., Eltayeb, H., Atan, K.A.M.: A note on the comparison between Laplace and Sumudu transforms. Bull. Iran Math. Soc. 37(1), 131-141 (2011)

19. Kilicman, A., Gadain, H.E.: On the applications of Laplace and Sumudu transforms. J. Franklin Inst. 347, 848-862 (2010)

20. Kumar, D., Choi, J., Srivastava, H.M.: Solution of a general family of fractional kinetic equations associated with the generalized Mittag-Leffler function. Nonlinear Funct. Anal. Appl. 23(3), 455-471 (2018)

21. Mathieu, E.L.: Traité de Physique Mathematique, VI-VII, Théorie de l'élasticité des Corps Solids. Gauthier-Villars, Paris (1980)

22. Nisar, K.S., Qi, F.: On salutation of fractional kinetic equations involving the generalized k-Bessel function. Note Mat. 37(2), 11-20 (2017)

23. Prabhakar, T.R.: A singular integral equation with a generalized Mittag-Leffler function in the kernel. Yokohama Math. J. 19, 7-15 (1971)

24. Saichev, A.I., Zaslavsky, G.M.: Fractional kinetic equations: solutions and applications. Chaos 7(4), 753-764 (1997)

25. Saxena, R.K., Kalla, S.L.: On the solutions of certain fractional kinetic equations. Appl. Math. Comput. 199, 504-511 (2008)

26. Saxena, R.K., Mathai, A.M., Haubold, H.J.: On fractional kinetic equations. Astrophys. Space Sci. 282, 281-287 (2002)

27. Saxena, R.K., Mathai, A.M., Haubold, H.J.: Unified fractional kinetic equations and a fractional diffusion equation. Astrophys. Space Sci. 290, 299-310 (2004)

28. Saxena, R.K., Mathai, A.M., Haubold, H.J.: On generalized fractional kinetic equations. Physica A 344, 657-664 (2004)

29. Saxena, R.K., Ram, J., Kumar, D.: Alternative derivation of generalized fractional kinetic equations. J. Fract. Calc. Appl. $4(2), 322-334(2013)$

30. Schiff, J.L.: The Laplace Transform: Theory and Applications. Springer, Berlin (1999)

31. Shaktawat, B.S., Gupta, R.K., Kumar, D.: Generalized fractional kinetic equations and its solutions involving generalized Mittag-Leffler function. J. Rajasthan Acad. Phys. Sci. 16, 63-74 (2017)

32. Spiegel, M.R.: Theory and Problems of Laplace Transforms. Schaums Outline Series. McGraw-Hill, New York (1965)

33. Srivastava, H.M., Saxena, R.K.: Operators of fractional integration and their applications. Appl. Math. Comput. 118, 1-52 (2001)

34. Srivastava, H.M., Tomovski, Z:: Some problems and solutions involving Mathieu' series and its generalizations, J. Inequal. Pure Appl. Math. 5(2), Article ID 45 (2004)

35. Tomovski, Z., Mehrez, M.: Some families of generalized Mathieu-type power series. Appl. Anal. Discrete Math. 20(4), 973-986 (2017)

36. Tomovski, Z., Pogány, T.K.: Integral expressions for Mathieu-type power series and for the Butzer-Flocke-Hauss function. Fract. Calc. Appl. Anal. 14(4), 623-634 (2011)

37. Tomovski, Z., Trencevski, K.: On an open problem of Bai-Ni Guo and Feng Qi. J. Inequal. Pure Appl. Math. 4(2), Article ID 29 (2003)

38. Watugala, G.K.: Sumudu transform: a new integral transform to solve differential equations and control engineering problems. Int. J. Math. Educ. Sci. Technol. 24(1), 35-43 (1998)

39. Watugala, G.K.: The Sumudu transform for function of two variables. Math. Eng. Ind. 8, 293-302 (2002)

40. Wiman, A.: Über den fundamental Satz in der Theorie der Funktionen $E_{\alpha}(X)$. Acta Math. 29, 191-201 (1995)

\section{Submit your manuscript to a SpringerOpen ${ }^{\circ}$ journal and benefit from:}

- Convenient online submission

- Rigorous peer review

- Open access: articles freely available online

- High visibility within the field

- Retaining the copyright to your article

Submit your next manuscript at $\gg$ springeropen.com 